Supporting Information for

\title{
Occupied and Unoccupied Levels of Half- and Per-fluorinated Rubrene Thin Films Probed by One- and Two-Photon Photoemission
}

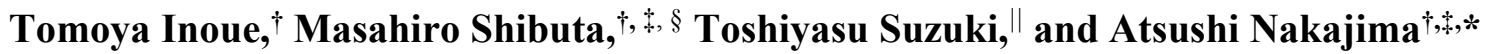 \\ ${ }^{\dagger}$ Department of Chemistry, Faculty of Science and Technology, and ${ }^{\ddagger}$ Keio Institute of Pure and Applied \\ Sciences (KiPAS), Keio University, 3-14-1 Hiyoshi, Kohoku-ku, Yokohama 223-8522, Japan \\ ${ }^{\S}$ Fachbereich Physik und Zentrum für Materialwissenschaften, Philipps-Universität, D-35032 Marburg, \\ Germany \\ "Instrument Center, Institute for Molecular Science, Myodaiji, Okazaki 444-8585, Japan \\ *Address correspondence to A. Nakajima \\ Tel: +81-45-566-1712, Fax: +81-45-566-1697, E-mail: nakajima@,chem.keio.ac.jp
}

Table of Contents

Figure S1. Determination of layer thickness.

(Page S2)

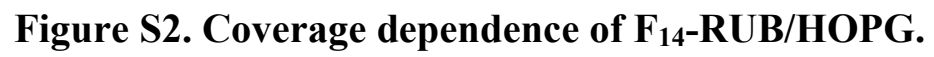

(Page S2)

Figure S3. Coverage dependence of PF-RUB/HOPG.

(Page S3)

Figure S4. XPS survey spectra of $F_{14}-R U B$ and PF-RUB on HOPG.

(Page S3)

Figure S5. C $1 s$ core-level XPS spectra of $\mathrm{F}_{14}-\mathrm{RUB}$ and PF-RUB on HOPG. $\quad$ (Page S4)

Figure S6. Coverage dependence of the vacuum level $\left(E_{V a c}\right)$ (Page S4)

Figure S7. Raw 2PPE spectra of the PF-RUB film (0.3 ML) on HOPG. $\quad$ (Page S5) 

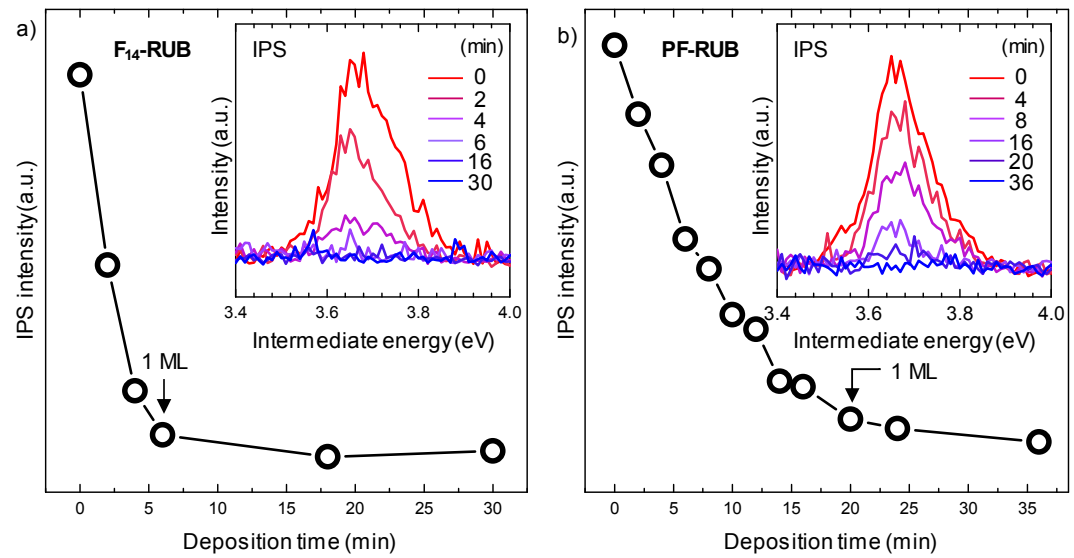

Figure S1. Determination of $\mathrm{F}_{14-\mathrm{RUB}}$ and PF-RUB coverages of HOPG, which are evaluated by $2 \mathrm{PPE}$ signal intensity of the image potential state (IPS) formed on the HOPG substrate. (a and b) IPS intensities against the deposition time $t$ of (a) F $_{14-R U B}$ and (b) PF-RUB. The IPS of the bare HOPG substrate is attenuated by the molecular depositions where the original 2PPE spectra around the IPS are displayed in insets. The IPS mostly vanishes at $t=6 \mathrm{~min}$ for F14-RUB or $t=20 \mathrm{~min}$ for PF-RUB, indicating that these values correspond to the times used to obtain a full coverage (1 ML) of $F_{14-R U B}$ or PF-RUB films. It should be noted that the deposition rate is not calibrated in the present deposition procedure and that we only confirm the stability of the deposition rate by quartz microbalance. The coverage of $\mathrm{F}_{14}-\mathrm{RUB}$ or PF-RUB is then controlled by $\mathrm{t}$, namely $\mathrm{ML}\left(\mathrm{F}_{14-\mathrm{RUB}}\right)=t / 6 \mathrm{~min}$ and $\mathrm{ML}$ (PF$\mathrm{RUB})=t / 20 \mathrm{~min}$.
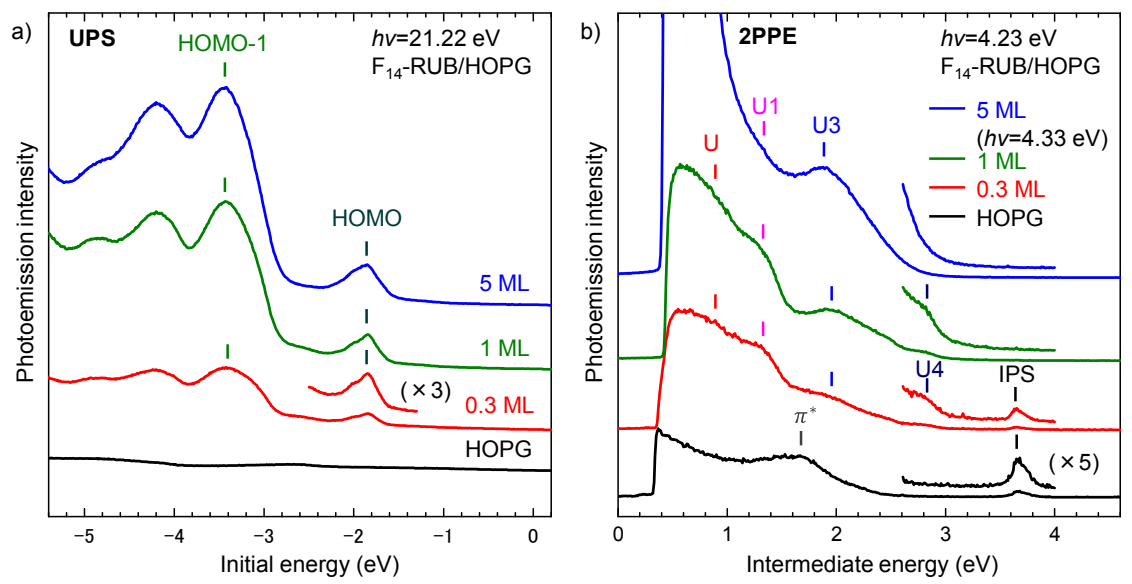

Figure S2. Coverage dependence of the (a) UPS spectra and (b) 2PPE spectra of F14-RUB films on HOPG. The coverages of the films are given on the right sides. In the UPS spectra, a series of occupied molecular orbitals (HOMO, HOMO-1, ...) is resolved. The HOMO-derived peak contains fine structures arising from vibronic couplings (see the main text), which become vague at higher coverages. The 2PPE spectra (b) show several spectral features, named as U, U1, U3, and U4 by the deposition of $\mathrm{F}_{14-R U B}$ while the IPS of HOPG is attenuated with an increase in the coverage (Figure S1). The spectral features of $\mathrm{U}-\mathrm{U} 4$ are assigned to be unoccupied levels. Judging from the $h v$ dependence of the 2PPE measurements (Figure 4), the photon energy is $4.23 \mathrm{eV}$ except for $5 \mathrm{ML}$ (measured at 4.33 $\mathrm{eV})$. 

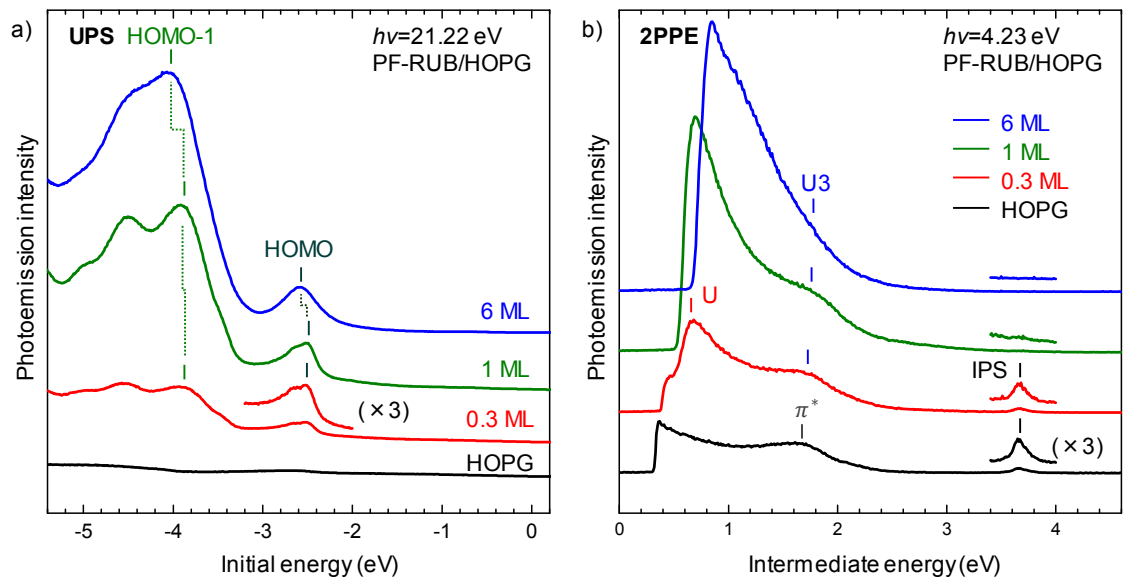

Figure S3. Coverage dependence of the (a) UPS spectra and (b) 2PPE spectra of PF-RUB films on HOPG. The coverage of the films is given on the right sides. In the UPS spectra, the series of occupied molecular orbitals (HOMO, HOMO-1, ...) appear. Similar to the F14-RUB case in Figure S2(a), the HOMO-derived peak contains fine structures with vibronic coupling at low coverage $(0.3 \mathrm{ML})$. The 2PPE spectra (b) show unoccupied $U$ and U3 structures at $0.3 \mathrm{ML}$, where both structures lose their significance at higher coverages with an increment in low-energy cutoff (U) (also see Figure S6) or signals of secondary electrons (U3).
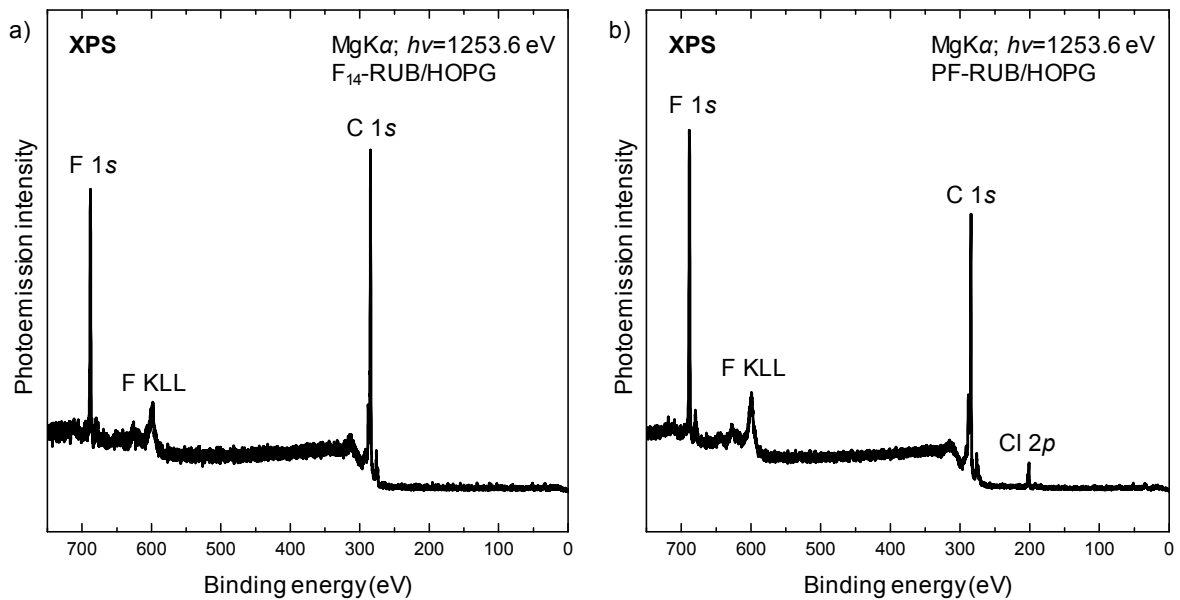

Figure S4. The XPS spectra (MgK $\alpha, h v=1253.6 \mathrm{eV}$ ) of (a) $\mathrm{F}_{14-\mathrm{RUB}}$ and (b) PF-RUB films (1 ML) on HOPG. Both spectra indicate that these molecular films mostly consist of only carbon and fluorine (and hydrogen) atoms, while the slight signal originating from residual chlorine atom is recognized in the PF-RUB film in (b). The C $1 s$ signal involves not only carbons from $\mathrm{F}_{14-R U B}$ or PF-RUB molecule but also carbons from the HOPG substrate as the main contribution (see Figure S5). 

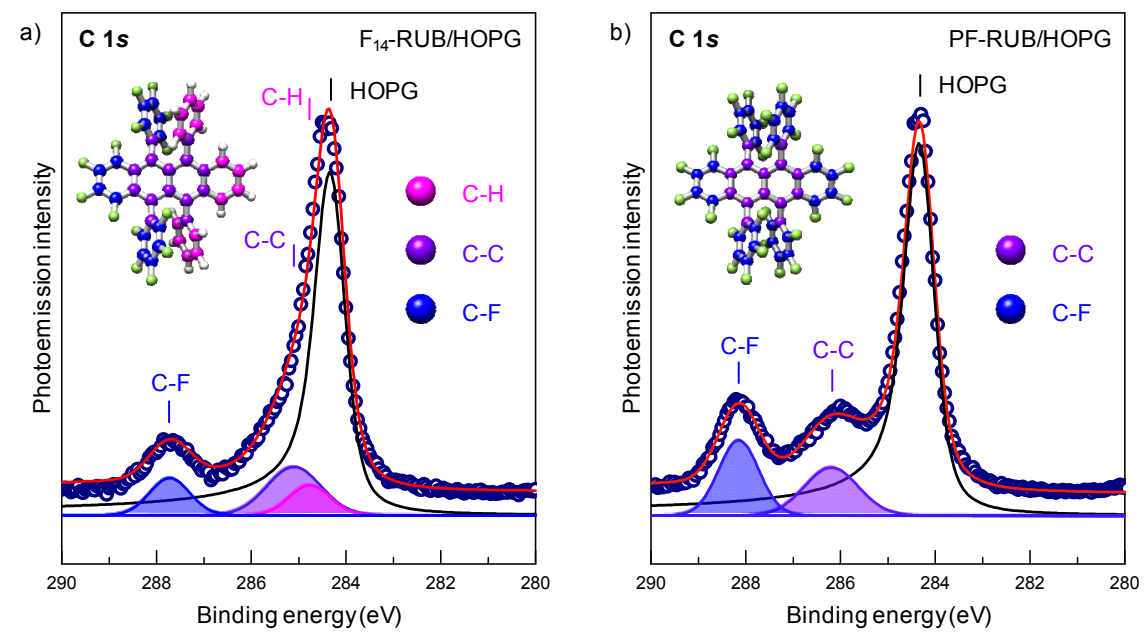

Figure S5. The XPS spectra in the vicinity of C $1 s$ core level of (a) F14-RUB and PF-RUB films in HOPG. In addition to the main peak derived from the HOPG substrate at the binding energy of 284.3 $\mathrm{eV}$, several peak components appear by $\mathrm{F}_{14}$-RUB or PF-RUB depositions. For a chemical analysis, at first, the $\mathrm{C} 1 s$ core levels of PF-RUB/HOPG is deconvoluted using three components: (1) HOPG, (2) carbon atoms forming $\mathrm{C}-\mathrm{C}$ bonds $(\mathrm{C}-\mathrm{C}$ carbon) at $286.2 \mathrm{eV}$ (purple-colored carbon atoms in inset (b)), and (3) carbon atoms forming $\mathrm{C}-\mathrm{F}$ bonds (C-F carbon) at $288.1 \mathrm{eV}$ (blue-colored carbon atoms). Based on the above analysis of PF-RUB/HOPG, the C $1 s$ core level are deconvoluted into four components: (1) HOPG, (2) C-C carbon at $285.1 \mathrm{eV}$, (3) $\mathrm{C}-\mathrm{F}$ carbon at $287.7 \mathrm{eV}$, and (4) carbon atoms forming $\mathrm{C}-\mathrm{H}$ bonds $(\mathrm{C}-\mathrm{H}$ carbon) at $284.8 \mathrm{eV}$ (pink-colored carbon atoms in inset (a)). The $\mathrm{C}$ $1 s$ peak positions with F14-RUB and PF-RUB obtained by the above fittings agree with those reported by Anger et al. ${ }^{1}$
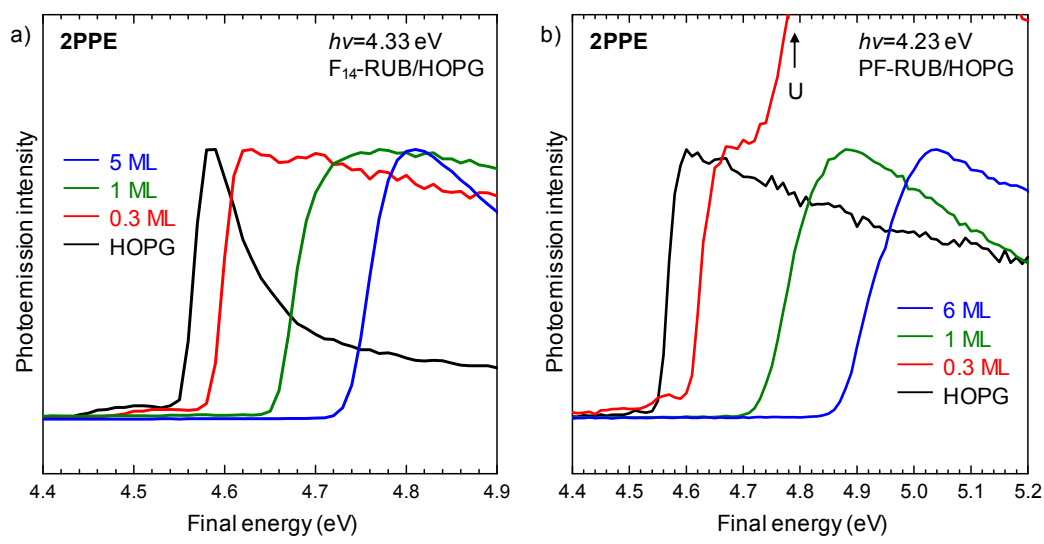

Figure S6. The coverage dependence of 2 PPE spectra of (a) F14-RUB and (b) PF-RUB films on HOPG around low-energy thresholds. All spectra are normalized with 2PPE intensity at the low-energy edge. The thresholds in the final energy plots correspond to $E_{\mathrm{Vac}}$ with respect to $E_{\mathrm{F}}$. The $E_{\mathrm{Vac}}$ increases in parallel with the F14-RUB and PF-RUB coverages: $4.58 \mathrm{eV}$ (0.3 ML), $4.66 \mathrm{eV}$ (1 ML), $4.74 \mathrm{eV}$ (5 ML) of F14-RUB and $4.61 \mathrm{eV}(0.3 \mathrm{ML}), 4.74 \mathrm{eV}$ (1 ML), $4.87 \mathrm{eV}$ (6 ML) of PF-RUB. A hump of 2PPE intensity in the spectrum of $0.3 \mathrm{ML}$ PF-RUB/HOPG is ascribed to the unoccupied level of U. 


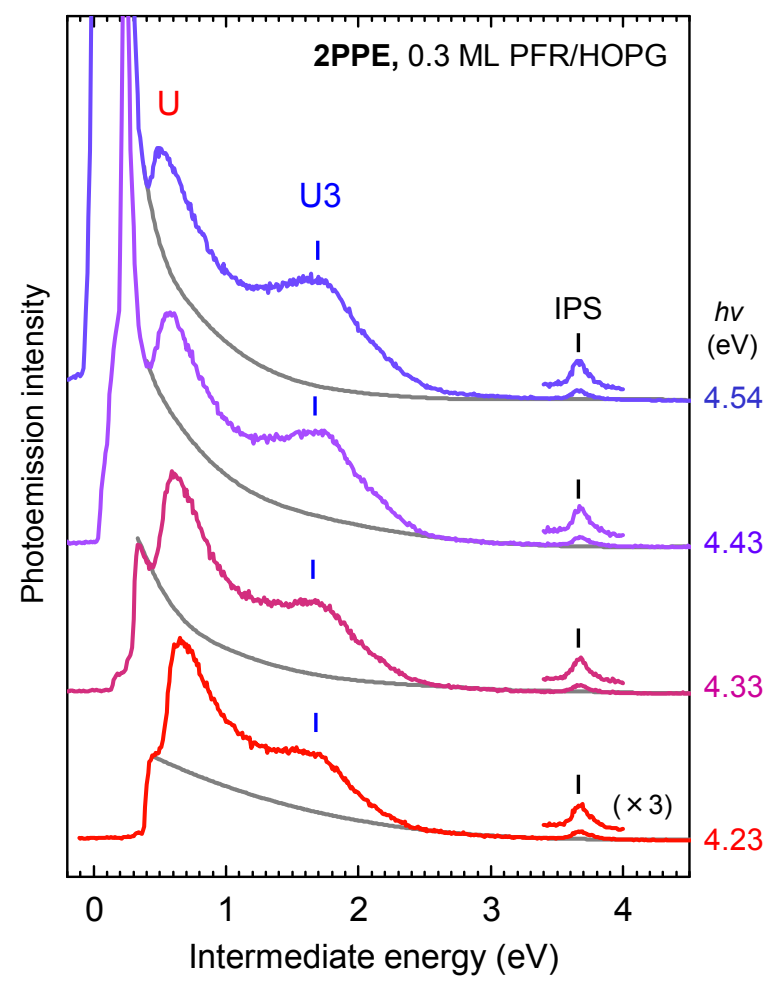

Figure S7. Original data of $h v$-dependent 2PPE spectra of 0.3 ML PF-RUB film on HOPG measured with $h v=4.23-4.54 \mathrm{eV}$. Although the $\mathrm{U}$ peak seemingly shifts toward the lower intermediate energy as a result of an increase in $h v$, it is considered that the peak energy is gravitated by the secondary electron signals as the background. Since the contribution of background signals becomes larger at higher $h v$, the low-energy cutoff simultaneously shifts toward lower energy. Then, the background profile is assumed as an exponential decay-like curve (gray solid line) where the subtracted 2PPE spectra are indicated in Figure 5(a). The $\mathrm{U}$ peak shows a constant intermediate energy as $0.6 \mathrm{eV}$, which is similar to that of U3 and IPS when removing the influence of the low-energy background (see Figure $5(\mathrm{a}))$.

\section{Reference}

(1) Anger, F.; Glowatzki, H.; Franco-Cañellas, A.; Bürker, C.; Gerlach, A.; Scholz, R.; Sakamoto, Y.; Suzuki, T.; Koch, N.; Schreiber, F. Interface Dipole and Growth Mode of Partially and Fully Fluorinated Rubrene on $\mathrm{Au}(111)$ and $\mathrm{Ag}(111)$, J. Phys. Chem. C. 2015, 119, 6769-6776. 